\title{
Design Considerations of a Low-Complexity, Low- Power Integer Turbo Decoder
}

\author{
Stephen M. Pisuk and Peter H. Wu \\ MIT Lincoln Laboratory, Lexington MA 02420
}

\begin{abstract}
In this paper we present an efficient Turbo decoder implementation with minimal performance loss. Based on the non-optimal Max-Log-MAP algorithm, we carefully manipulate the algorithm, through a-priori scaling to improve BER within only $0.25 \mathrm{~dB}$ loss of $\mathrm{E}_{b} / \mathrm{N}_{0}$ of the optimal decoder. All computations are performed as integers avoiding the complexity and logic requirements of fixed and floating-point arithmetic. By combining integer computations with a design based on a single "sliding-window" decoder and a zero-delay interleaver, the final FPGA implementation achieves $1 \mathrm{Mbps}$ while consuming less than $600 \mathrm{~mW}$.
\end{abstract}

\section{INTRODUCTION}

Turbo coding has shown great promise with near channel capacity achieved with long block sizes and numerous decoding iterations [1]. However the enormous computational complexity required for the decoder has thus far limited the application of these codes. Nevertheless, several upcoming communication systems (e.g. IMT-200 and MILSTAR Advanced EHF) have chosen to utilize the coding gain of Turbo codes.

In order to truly utilize Turbo codes, one must find an efficient decoder implementation in terms of both BER and computational resources. This is particularly true in wireless systems as well as any portable application where size and power are key limitations. The goal of reduced size and power require simplification of the decoding algorithm as well as a careful and compact hardware implementation.

This work is sponsored by the Department of the Air Force under contract F19628-00-C-0002. Opinions, interpretations, conclusions are those of the author and not necessarily endorsed by the United States Government. 
The optimal Turbo decoding algorithm is Log-MAP[2]. Its complexity arises from a modification of the traditional Viterbi Add-Compare-Select (ACS) operation. In Log-Map, the Compare-Select has been replaced by $\max ^{*}(\mathrm{x}, \mathrm{y})=\ln \left(\mathrm{e}^{\mathrm{x}}+\mathrm{e}^{\mathrm{y}}\right)$. Through some manipulation, the $\max *(\mathrm{x}, \mathrm{y})$ function can be expressed as $\max *(x, y)=\max (x, y)+\ln \left(1+e^{-|x-y|}\right)$. From this alternate form of max*, two forms of simplification arise. The first form of simplification is to pre-compute the natural logarithm term and store the results in a look-up table[2]. However many tables are required for the design to be robust with a range of SNR. This is less complex than the straightforward Log-Map algorithm, but still too computationally intense for a low-power design. Along the same lines is the Linear-Log-Map algorithm[3]. Here the $\ln \left(1+e^{-|x-y|}\right)$ is approximated by a linear function. Linear-Log-Map accounts for the fact that the entire term is approximately 0 when $|x-y|$ is greater than 3 or 4 . This achieves near optimal performance and reduced complexity. In order to truly simplify the Turbo decoding algorithm, we come to the second class of simplifications to the max* function. Algorithms such as Max-Log-MAP [2] approximate the $\max *(x, y)$ with $\max (\mathrm{x}, \mathrm{y})$. Although this results in approximately $0.5 \mathrm{~dB}$ of performance loss, the computational requirements are greatly reduced. In addition, by slightly modifying the Max-Log-MAP algorithm, we are able to gain back a portion of the $0.5 \mathrm{~dB}$ loss while still realizing a tremendous reduction in computational complexity.

Although many fixed and floating-point decoder implementations have been presented[3][4][5][6], these implementations appear to be too complex. Although less complex than floating-point, fixed-point arithmetic still requires normalization. We constrain ourselves to 2's compliment integer representations for all calculations and metric storage. While we still must consider the dynamic range of our numbers, integer normalization does not require multiplication or division.

In addition, we have further simplified our decoding process by implementing a single decoding engine rather than a traditional two-decoder design. Although this will be further explored in Section 2.4, this simplification allows to the effectively reduce the hardware resources by a factor of two.

For reference, the specifications of the coding scheme presented are as follows:

- Code Rate $1 / 2$

- Constituent code $(23,35)_{\text {octal }}$

- Odd/Even Puncturing

- Block Size 640 with no trellis termination

- S=18 S-Random Interleaver 


\section{IMPLEMENTATION SPECIFICS}

\subsection{Turbo Decoder Basics}

Traditional Turbo decoders utilize two decoding engines separated by a set of interleavers and de-interleavers. Each decoder inputs a set of noisy codewords $\left\{\mathrm{y}_{\mathrm{k}}{ }^{1}, \ldots, \mathrm{y}_{\mathrm{k}}{ }^{1 / \mathrm{R}}\right\}$ ( $\mathrm{R}$ is the code-rate) as well as an a-priori value $\mathrm{La}_{\mathrm{k}}$. After some processing interval, the decoder produces soft-decisions, $\mathrm{Lu}_{\mathrm{k}}$, and extrinsic information, $\mathrm{Le}_{\mathrm{k}}$. The extrinsic information output from one decoder is passed through an interleaver or de-interleaver and becomes the a-priori input to the next decoder. As shown in Figure $1,\{\mathrm{Le}\}_{\mathrm{D} 1}$, the extrinsic output from decoder 1 , is passed through an interleaver and becomes $\{\mathrm{La}\}_{\mathrm{D} 2}$ the a-priori input to decoder 2. Similarly, $\{\mathrm{Le}\}_{\mathrm{D} 2}$ is deinterleaved and becomes $\{\mathrm{La}\}_{\mathrm{D} 1}$ for the next decoding iteration.

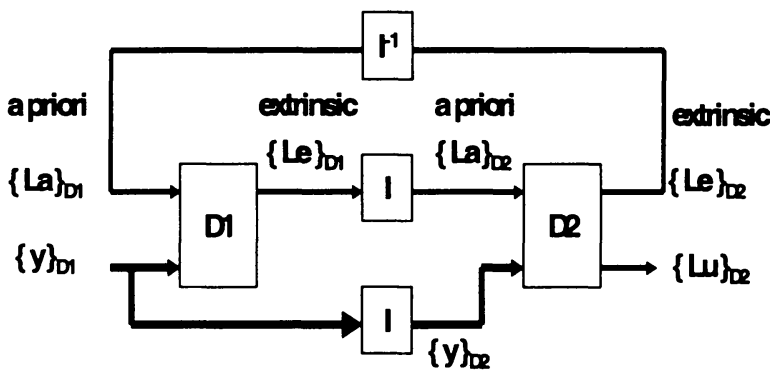

Figure 1. Traditional Decoder Design

This process of exchanging information is what produces the coding gain present in Turbo coding. Because each decoder has a different view of the data, it can offer insight to the other decoder not otherwise possible. This exchange of information continues for a number of iterations at which point the soft decisions are limited to form the final hard decision. More generally, this process is known as iterative decoding.

For a more complete theoretical explanation of the max-Log-MAP algorithm please refer to [2] or [9]. 


\subsection{Modified Max-Log-MAP}

In our modified Max-Log-Map decoder, we carefully examine the computation of the branch metric. The equation is as follows:

$$
c_{k}\left(s^{\prime}, s\right)=\left(\frac{1}{2}\right)\left\{\sum_{j=1}^{n} y_{k}^{(j)} \cdot x_{k}^{(j)}\left(s^{\prime}, s\right)+L a_{k} \cdot u_{k}\left(s^{\prime}, s\right)\right\}
$$

In the first part of the equation, the incoming noisy codewords $y_{k}$ are correlated against the $\mathrm{j}$ possible codewords, $\mathrm{x}_{\mathrm{k}}$, generated by the encoder. The second part of the equation represents the a-priori knowledge about this transition from all previous decoders. For the first decoder of the first iteration, the a-priori is set to 0 , as we do not have any a-priori knowledge. Essentially we are combining past decoding results with the current decoding results to form a new result.

By adding a scaling constant in front of either term, we can control the strength of the current result against the past result. For example, if we were to multiply the codeword correlation by a number greater than 1 , intuitively we are saying that the result from the previous decoders are less important than the current decoder's results. Similarly, if we multiply by a number less than one, we are stating that the previous decoder's result should be valued more than the current result. Hence we arrive at the following equation:

$$
c_{k}\left(s^{\prime}, s\right)=\left(\frac{1}{2}\right)\left\{\sum_{j=1}^{n} y_{k}^{(j)} \cdot x_{k}^{(j)}\left(s^{\prime}, s\right)+R \cdot L a_{k} \cdot u_{k}\left(s^{\prime}, s\right)\right\}
$$

We have added a scaling constant $\mathrm{R}$ to the a-priori information. As verified in [7], if SNR is constant and $R$ is swept from 0 to 2, thus varying the importance of the a-priori information, a wide range of BER is observed. 


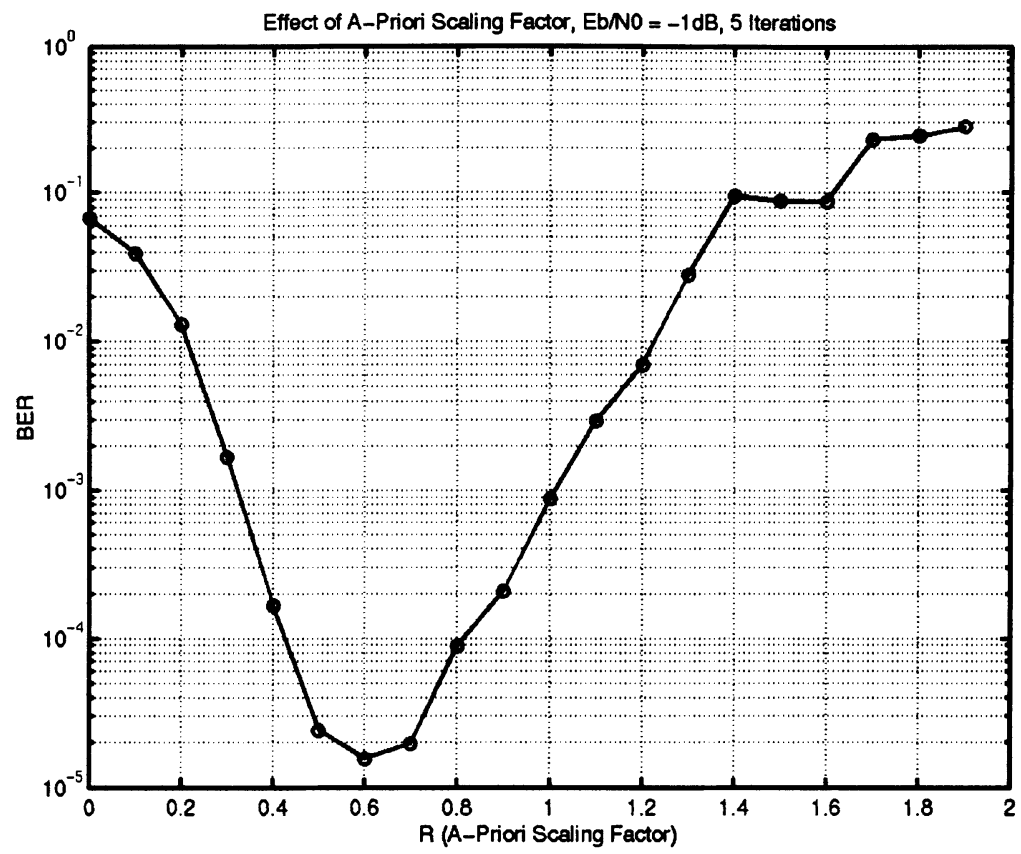

Figure 2. BER vs. A-Priori Scaling Constant

As Figure 2 shows, when $0.45<=\mathrm{R}<=0.6$ the BER is improved by an order of magnitude. By setting $R=0.5$, we are able to gain back $0.35 \mathrm{~dB}$ of the approximate $0.5 \mathrm{~dB}$ loss incurred by using Max-Log-MAP instead of the optimal Log-MAP.

Although the absolutes reason for the BER improvement cannot be totally explained, [7][9] suggest that $\mathrm{R}$ mitigates the propagation of errors from iteration to iteration. Normally a cluster of errors passed to the next decoder via extrinsic information dominates the branch metric calculations in the decoder. More generally, the codeword correlation is not strong enough to right an extreme error in the a-priori information serving to propagate errors.

\subsection{Sliding-Window Decoding}

As explained in [1][2][9], Log-MAP decoding is broken into two main components, forward recursion and backward recursion. Forward recursion steps through the trellis from bits 0 to $\mathrm{N}-1$ producing node metrics representing the logarithmic probabilities that the encoder was in a given state. Backward recursion has two tasks. First it calculates node retrics like 
forward recursion, but steps its way through the trellis from bit $\mathrm{N}-1$ to bit 0 . Second, it combines the probability of arriving at a node via forward recursion with the probability of arriving at the next node via backward recursion into a complete path metric. The complete path metrics for a bit may be combined to form a soft decision of the transmitted bit.

Unfortunately, traditional Log-MAP-based decoders require large amounts of memory because the decoder must store all forward node metrics as well as all branch metrics for use in backward recursion. For the decoder presented here: 8 bits per forward node metric, 16 node metrics per bit, 640 bits per decoder block, or 81,920 bits. For branch metrics, 6 bits per branch metric, 8 branch metrics per bit, 640 bits per decoder, or 30,720 bits. This is a prohibitively large memory requirement for a single FPGA implementation. To combat this problem, sliding-window or sub-block processing was proposed in [8].

With sliding-window processing, the backward recursion unit is split into a backward training metric generator, and a backward soft-decision generator (shown in Figure 3).

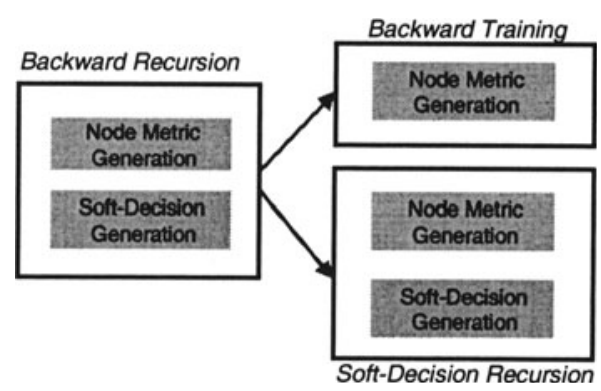

Figure 3. Sliding-Window Modifications

According to [8], after 5 to 6 times the constraint length, backward metrics are considered reliable. This allows us to decode the code block in segments rather than as a whole. Conveniently, the sliding-window block was chosen to be 32 bits long for constraint length 5 . This result is that the decoder only needs to store 64 bits worth of forward node metrics and branch metrics rather than 640 bits worth. This reduces the memory requirements by a factor of 10 .

In sliding-window processing, the backward soft-decision recursion unit is exactly the same as the backward recursion unit in traditional Log-MAP processing. The backward training metric generator is a stripped down version of the backward soft-decision generator, as it only contains the nodemetric generation logic. 
Figure 4 shows a brief example of how sliding-window processing works. $\mathrm{L}$ is equal to 5 to 6 times the constraint length. As the forward recursion unit works from $\mathrm{L}$ to $2 \mathrm{~L}$, the backward training metric generator works from $3 \mathrm{~L}$ back to $2 \mathrm{~L}$.

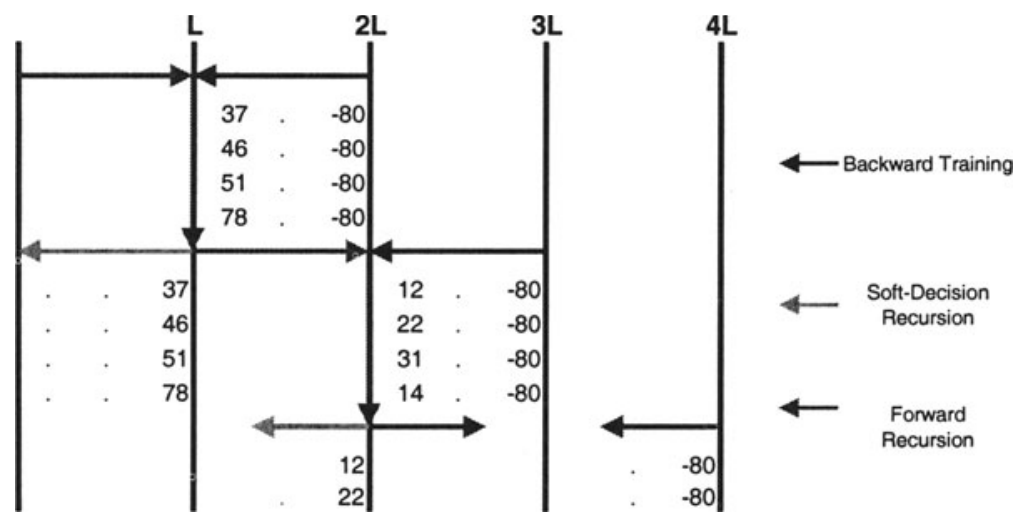

Figure 4. Sliding-Window Processing

When the two units meet, the backward training metric generator passes its node metrics to the soft-decision generator. The soft-decision generator then continues from $2 \mathrm{~L}$ back to $\mathrm{L}$ producing soft-decisions while the backward training metric generator processes $4 \mathrm{~L}$ down to $3 \mathrm{~L}$. This process is repeated until the entire decoder block is finished.

\subsection{Single Decoding Engine}

As shown in Figure 1, the traditional Turbo decoder utilizes two decoding engines separated by interleavers and de-interleavers. Because most Turbo codes utilize some sort of random interleavers for performance reasons, we cannot make any assumption about when bits will be ready. For example, if block or convolutional interleaver were used, we might be able to say that after D1 runs for $\mathrm{N}$ bits we can start D2. This is a nearly impossible assumption to make with random interleavers.

As a result, we simplify the decoder in Figure 1 to the decoder shown in Figure 5. 


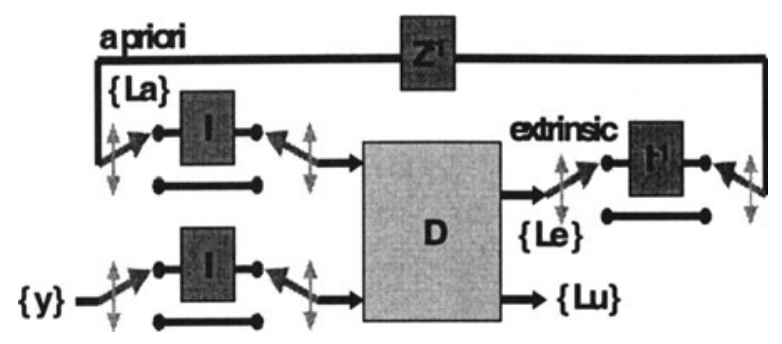

Figure 5. Single Decoder Design

Decoders D1 and D2 have been simplified into a single decoder D. The interleavers and de-interleavers remain, but multiplexers have been added to allow them to be bypassed. For example, if we are using D1, the interleaver and de-interleaver are not used so the multiplexers are set to the bypass position. However before beginning what was D2, the multiplexers are switched to pass the data into the interleavers and de-interleavers. In essence, we have traded an entire decoding engine for three multiplexers. This simplification results in an approximate $45 \%$ savings in area.

\section{$2.5 \quad$ Zero-Delay Interleaving}

Another aspect of the decoder design is its zero-delay interleaving. Turbo decoding requires interleavers on the inputs of D2 and a de-interleaver on the output of D2 in order to give D2 an alternate view of the data stream.

One way to accomplish this is to have separate interleaving and deinterleaving units that require some processing time to complete. With this approach, upon the completion of D1, its output would be interleaved and then fed into D2. Similarly when D2 was complete, its output would be deinterleaved and fed back into D1. However this approach would consume at least $\mathrm{N}$ additional clock cycles for interleaving and an additional $\mathrm{N}$ clock cycles for de-interleaving. Because additional processing time relates to increased power consumption, it is desirable to limit the time required by these functions.

The time required to interleave and de-interleave can effectively be reduced to zero if this process is performed by address manipulation. This is illustrated in Figure 6. 


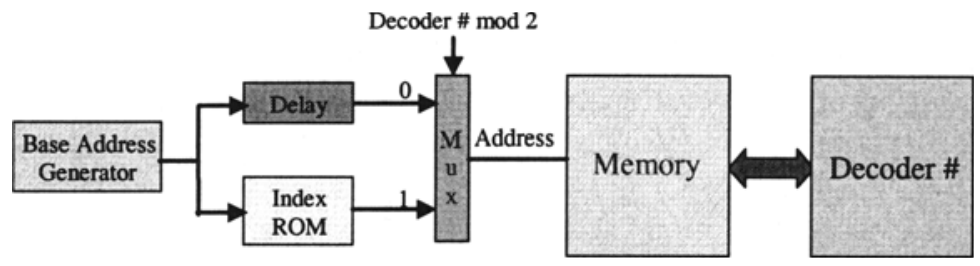

Figure 6. Address-based Interleaving

In the address manipulation approach, the base address generator simply counts from 0 to $\mathrm{N}-1$, where $\mathrm{N}$ is the block size of the code. If we are in the non-permuted decoder, D1, this count is simply delayed by a cycle and then used to read data out of input memory. However if we are in D2, the permuted decoder, the count is used to read an address from an Index ROM containing the interleaver sequence. The result from the ROM is then used to read from the input memory. As for de-interleaving, we simply pass the multiplexed address to the backwards soft-decision unit where the address is used to store the result.

Figure 7 illustrates the two interleaving schemes. In the first illustration, we see the traditional approach with distinct interleaving and de-interleaving stages. Although the implementation may be efficient, it will still require some finite processing interval. However when we examine the second illustration representing interleaving by address manipulation, we do not see any distinct interleaving blocks.
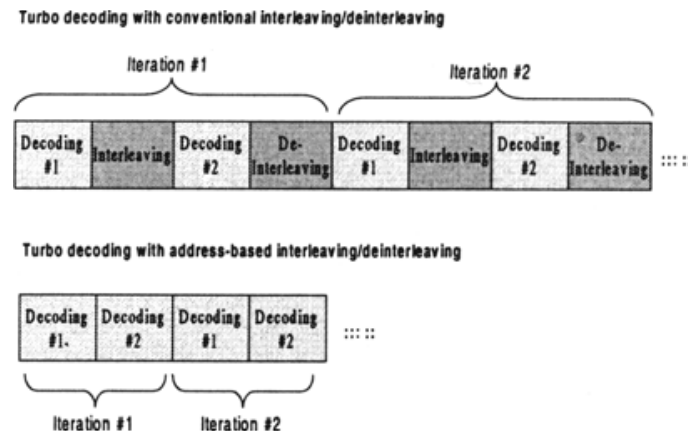

Figure 7. Various Interleaving Schemes

Because the interleaving / de-interleaving process has been combined with the normal process of retrieving data from memory, we are able to replace at least $\mathrm{N}$ cycles of interleaving delay by a clock cycle delay. 
At first this modification may seem minor. However let us consider the case where interleaving and de-interleaving both require $\mathrm{N}$ clock cycles. If the decoder runs for I iterations, the traditional approach spends $\mathrm{I}^{*} \mathrm{~N}$ cycles interleaving and $\mathrm{I}^{*} \mathrm{~N}$ cycles de-interleaving. Whereas the address manipulation scheme spends $2 * I$ cycles interleaving and zero cycles deinterleaving. For the decoder presented here with 5 iterations, this is a savings of approximately 6,390 clock cycles.

\subsection{Metric Normalization}

As shown in [2] and [3], forward/backward node metrics accumulate as recursion progresses. Because finite precision is used, the node metrics can easily overflow or underflow. This is especially catastrophic with 2's compliment integer representations where an overflow results in a negative number and an underflow results in a positive number. The overflow/underflow problem can be solved by metric normalization. For each decoded bit in both forward and backward recursion, all node metrics are compared with a threshold $\mathrm{T}$. If the absolute value of any node metric is greater than $\mathrm{T}$, then all node metrics are shifted towards the center, as shown in Figure 8.

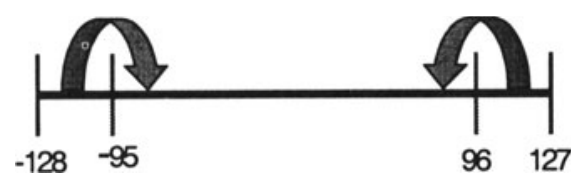

Figure 8. Forward/Backward Metric Normalization

Note that the shifting is done for all node metrics so that soft-decision values [10] are not affected. The threshold value is chosen such that the update (the node metric plus a branch metric) does not cause overflow and the logic required for comparison is minimized. In our implementation, the thresholds are set to be -95 and 96 . With normalization the node metrics range from -128 to 127 , which can be represented by 8 bits.

\section{RESULTS}

The Turbo Decoder was designed as a Verilog RTL description and implemented on a XILINX XCV600E-7 FPGA. The design utilized apprcximately 2900 out of 15550 Logic Elements (LEs) and approximately 24 out of 72 RAM blocks. The interleaver ROM is included and there are no external storage or processing components required. 
The design requires 1350 clock cycles per iteration with a maximum clock rate of approximately $25 \mathrm{MHz}$. When run at its normal operating point of 7 decoding iterations, this equates to approximately 1.7 megabits per second. At $1 \mathrm{Mbps}$, the decoder consumes less than $600 \mathrm{~mW}$

Figure 9 shows the BER performance of our all-integer FPGA Turbo decoder. Our 8-level integer-based Turbo decoder even outperforms floating point Max-Log-MAP, thanks to the a-priori scaling factor. Compared to the optimal (floating-point Log-MAP), the performance loss is less than $0.25 \mathrm{~dB}$ at $\mathrm{BER}=10^{-5}$.

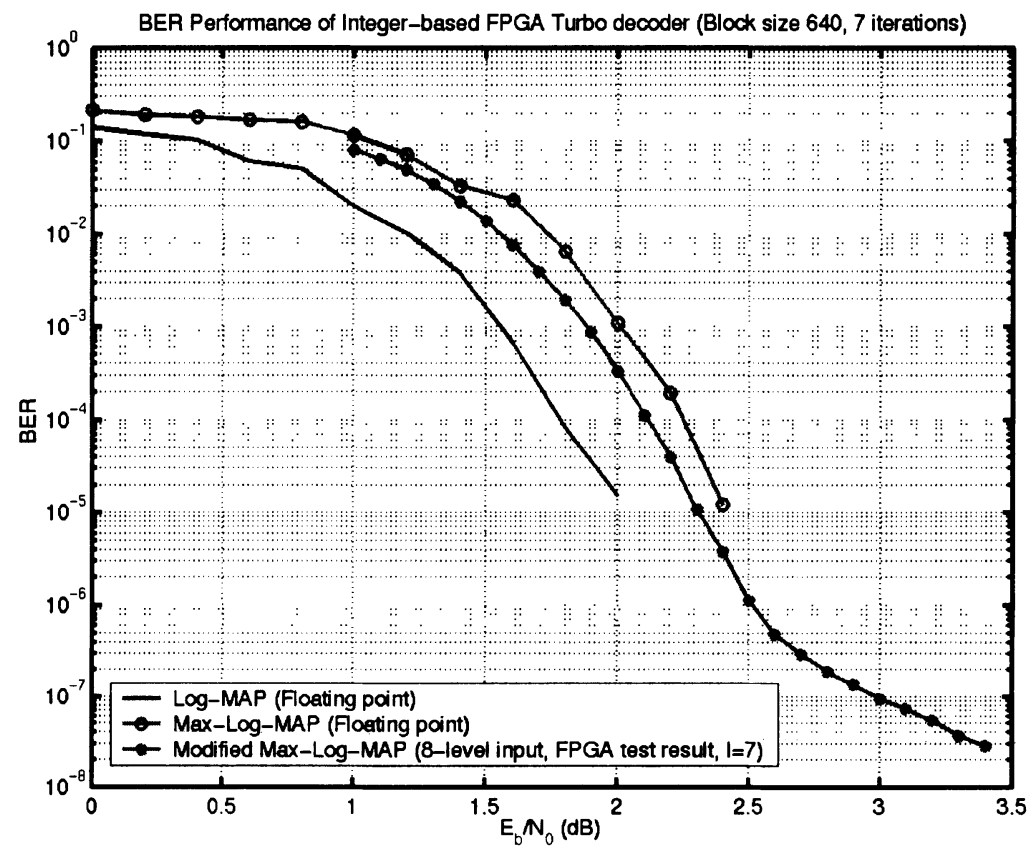

Figure 9. BER Performance of the integer FPGA Turbo Decoder (Block side $=640, S=18$ Interleaver, A-priori factor 0.5)

\section{CONCLUSION}

This paper presented the implementation of an integer Turbo decoder with minor performance loss. The efficient implementation comes from algorithm modification, integer arithmetic, and hardware management. Based on the Max-Log-MAP decoding algorithm, we modify the branch metric by weighting a-priori value, resulting in a significant BER improvement. 
By performing all computations as integers, the complexity of fixed and floating-point arithmetic was avoided. By analyzing the data flow through the interleavers we were able to simplify the traditional two-decoder design to a single decoder realizing a $45 \%$ savings in area. Interleaving was accomplished through address manipulation eliminating latency and resulting in higher throughput and lower power consumption. Finally, a simple metric normalization scheme was implemented to eliminate the possibility of overflow or underflow.

In summary, FPGA design utilizes approximately 2900 XILINX Logic Cells and consumes approximately $600 \mathrm{~mW}$ to achieve throughput of more than 1 Mbps. With 3 bits (8-level) input, our integer-based Turbo decoder outperforms floating-point Max-Log-MAP, and is only $0.25 \mathrm{~dB}$ away from the optimal floating-point Log-MAP.

\section{REFERENCES}

[1]C. Berrou, A. Glavieux, P. Thitimajshima, "Near Shannon limit error-correcting coding and decoding: Turbo Codes, " Proceedings of the 1993 International Conference on Communications, pp. 1064-1070, 1993.

[2]P. Robertson, E. Villebrun, and P. Hoeher, "A comparison of optimal and suboptimal MAP decoding algorithms operating in the Log domain," Proceedings of IEEE ICC '95, pp.1009-1013, June 1995.

[3] J.-F. Cheng and T. Ottosson, "Linearly Approximated Log-MAP Algorithms for Turbo Decoding," Proceedings of IEEE Vehicle Technology Conference 2000 Spring, pp. 22522256, May 2000.

[4] G. Montorsi and S. Benedetto, "Design of fixed-point iterative decoders for concatenated codes with interleavers," Proceedings of IEEE Globecom '00, Nov. 2000.

[5]G. Park, S. Yoon, C. Kang, D. Hong, "An implementation method of a Turbo-code decoder using a block-wise MAP algorithm," Proceedings of IEEE VTC '00 Fall, Sept. 2000.

[6]A. Wiesler, O. Muller, F. Jondral, "MAP-algorithm with fixed-point representation for software radios," IEEE VTC '00 Fall, Sept. 2000.

[7]J. Vogt and A. Finger, "Improving the max-log-MAP turbo decoder ," IEE Electronics Letters, Vol. 36, No. 23, Nov. 2000, pp.1937-1939.

[8]A. J. Viterbi, "An intuitive justification and a simplified implementation of the MAP decoder for convolutional codes," IEEE Journal on Selected Areas in Communications, VOL. 16, NO. 2, Feb. 1998, pp.260-264.

[9]P.H. Wu and S. Pisuk, "Implementation of a Low Complexity, Low-Power, Integer-based Turbo Decoder," Proceedings of IEEE Globecom ' 01, Nov. 2001.

[10] G. Battail, "Ponderation des symboles decodes par l'algorithm de Viterbi," Ann., Telecommun., vol. 42, pp. 31-38, Jan./Feb. 1987. 